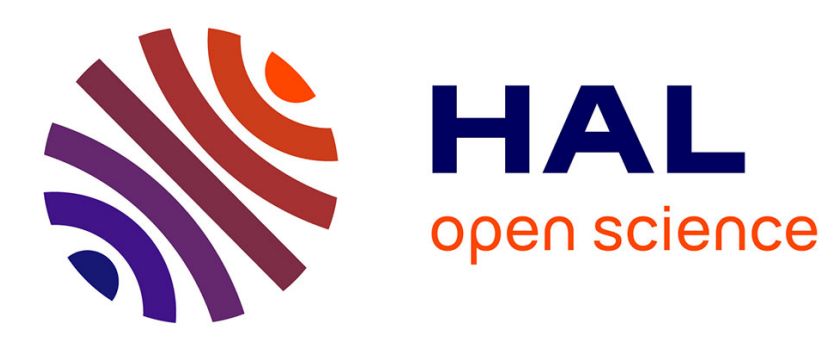

\title{
Characterization of Bubble Shapes in Non-Newtonian Fluids by Parametric Equations
}

Feishi Xu, Noel Midoux, Huai-Zhi Li, Gilles Hébrard, Nicolas Dietrich

\section{To cite this version:}

Feishi Xu, Noel Midoux, Huai-Zhi Li, Gilles Hébrard, Nicolas Dietrich. Characterization of Bubble Shapes in Non-Newtonian Fluids by Parametric Equations. Chemical Engineering and Technology, 2019, 42 (11), pp.2321-2330. 10.1002/ceat.201800690 . hal-02274472

HAL Id: hal-02274472

https://hal.science/hal-02274472

Submitted on 22 Nov 2019

HAL is a multi-disciplinary open access archive for the deposit and dissemination of scientific research documents, whether they are published or not. The documents may come from teaching and research institutions in France or abroad, or from public or private research centers.
L'archive ouverte pluridisciplinaire HAL, est destinée au dépôt et à la diffusion de documents scientifiques de niveau recherche, publiés ou non, émanant des établissements d'enseignement et de recherche français ou étrangers, des laboratoires publics ou privés. 


\section{CHARACTERIZATION OF BUBBLE SHAPES IN NON-NEWTONIAN FLUIDS BY} PARAMETRIC EQUATIONS

\section{Feishi Xu' ${ }^{1}$, Noel Midoux ${ }^{2}$, Huai-Zhi Li ${ }^{2}$, Gilles Hébrard ${ }^{1}$ and Nicolas Dietrich ${ }^{1, *}$}

${ }^{1}$ Laboratoire d'Ingénierie des Systèmes Biologiques et des Procédés (LISBP), Université de Toulouse, CNRS, INRA, INSA, 135 Avenue de Rangueil, 31077 Toulouse, France

'Laboratoire Réactions et Génie des Procédés (LRGP), Université de Lorraine, CNRS, 1 rue Grandville, BP 20451, 54001 Nancy Cedex, France

Email corresponding author: dietrich@insa-toulouse.fr

\section{Abstract}

Based on the experiment of single air bubbles rising in stagnant non-Newtonian fluids, an innovative model containing the aspect ratio $(E)$ and two parameters $(\alpha, \beta)$ was proposed and proved to be capable of characterizing the bubble shape from spherical/ellipsoidal to prolate/oblate-tear with good accuracy. Several impacts on bubble deformation were investigated involving the rheological properties of the fluids and different forces exerted on the bubble which were quantified by multiple dimensionless numbers (e.g. Reynolds number, Eötvös Number, Deborah number). Within a wide range ( $-9<\log M o<3$, Mo: Morton number), the empirical correlations were obtained for parameter $\beta$, and between $\alpha$ and $\beta$. Together with the shape model, a complete system was set up for bubble shape characterization and prediction that will provide new ideas for future studies on bubble hydrodynamics.

\section{Keywords: Bubble shape, Bubble dynamics, Gas-liquid system, Non-Newtonian fluid}

\section{Introduction}

Bubble-liquid systems are widespread in industries such as oil extraction, wastewater treatment and bioreactors. For these applications, the knowledge of bubble shape is of importance since it is directly related to hydrodynamic behavior (e.g. terminal velocity, drag, surface area) and can influence the mixing level and the efficiency of energy, mass, and momentum transfer.

The observed shapes of individual bubbles in free motion in Newtonian fluids have been well investigated and can be divided mainly into three categories: spherical, ellipsoidal, and spherical-cap or ellipsoidal-cap [1,2]. Grace et al. [3] have proposed a generalized graphical correlation or the so-called "Grace diagram" that delineates the shapes of bubbles in Newtonian fluids, which provides a guideline for the further studies on the bubble shape [4-6]. 
On the other hand, although numerous studies deal with bubbles rising in non-Newtonian media, only a few of them have discussed and analyzed the bubble shape [7-12]. It is generally agreed that non-Newtonian fluids have complex rheological properties (e.g. shear thinning, viscoelasticity, etc.) leading to a variety of bubble shapes, such as teardrop or elongate bubbles [13-18].

To quantitatively characterize the bubble shape, many researchers used the aspect ratio or eccentricity i.e. the ratio between the lengths of the major and minor axes of the bubbles. Pioneering work by Moore [19] and Tadaki and Maeda [20] theoretically correlated the aspect ratio of the bubble with the Weber number and original Tadaki number $T a=$ Re. $\left.M o^{0.23}\right)$, respectively. Their correlation were modified and improved for small inertial effects [21] and became applicable to clean and contaminated bubbles [22-24] for a large range of Morton numbers [25]. More recently, many researchers have begun to realize that, for fluids with high viscosity, no single dimensionless number (Eo, We, or Ta number) is suitable to predict the bubble deformation. Therefore, more dimensionless numbers have been used and many empirical models have been built to fit the results of the new experiments or numerical simulations [25-28]. For instance, Liu et al. [29] suggested that, in highly viscous fluids, the bubble shape is dramatically affected by the viscosity and the effects of We should be considered along with Re. However, the aspect ratio suffers from the shortcoming of being unable to characterize the details of shape or irregular forms (e.g. a cap bubble and an ellipsoidal bubble would have the same aspect ratio). A few researchers have tried to use other parameters to characterize the bubble deformation. Myint et al. [30] introduced a distortion factor to quantify the asymmetry between the upper and lower parts of the bubble divided by the major axis. Another study, by Yan and Zhao [31], used the bubble circularity, defined as the ratio of the circumference of an equivalent circle to that of the real bubble projection. Although some valuable conclusions have been drawn from the previous work, most of the expressions mentioned above could only handle quasi-ellipsoidal bubbles. For a bubble with a more complicated contour (e.g. cap, teardrop), it is clear that neither the aspect ratio nor the circularity can properly characterize the bubble shape.

Therefore, in this paper, a new model using the parametric equations is proposed to directly depict the complete profile of the bubble. Experiments will be implemented to study individual air bubbles of different sizes (equivalent diameters: $0.7-7 \mathrm{~mm}$ ) rising in stagnant nonNewtonian fluids (aqueous solution of polyacrylamide or Xanthan gum) by means of highspeed photography and image post-processing technologies. The objective mainly aims to investigate the overall impact (including the different dimensionless numbers and the rheological properties of the fluid) on the bubble shape. 


\section{$2 \quad$ Materials and method}

73

74

75

76

77

78

79

80

81

82

83

84

85

86

87

88

89

90

91

92

93

94

95

96

97

\subsection{Experimental setup}

The experimental setup for studying bubble shapes is shown in Figure 1. A column (volume: $100^{*} 100^{*} 300 \mathrm{~mm}^{3}$ ) was filled with $2 \mathrm{~L}$ of the liquid under study. A single bubble was generated by injecting the air into the column through a horizontal nozzle located $20 \mathrm{~mm}$ above the column bottom by a syringe pump (Harvard Apparatus, PHD 22/2000) with the flow rate of $10 \mu \mathrm{L} / \mathrm{h}$. Stainless steel nozzles of different calibers $(\approx 0.5-1 \mathrm{~mm})$ were used to make bubbles of different sizes (equivalent diameters: 0.7-7 mm). A high-speed camera (Photon SA3, resolution: $1024 \times 1024$ pixels) was placed next to one side of the column and focused on the rising bubble with a vertical image area $\left(\approx 40 \times 40 \mathrm{~mm}^{2}\right)$. The recording rate of the bubble images was 2000 frames per second. The image area was illuminated by a backlight board placed at the opposite side of the column. The images acquired by the camera were transmitted to the computer and processed with a professional software (PFVPhotron FASTCAM Viewer). All the experiments were performed at $293.15 \mathrm{~K}$ and under atmospheric pressure.

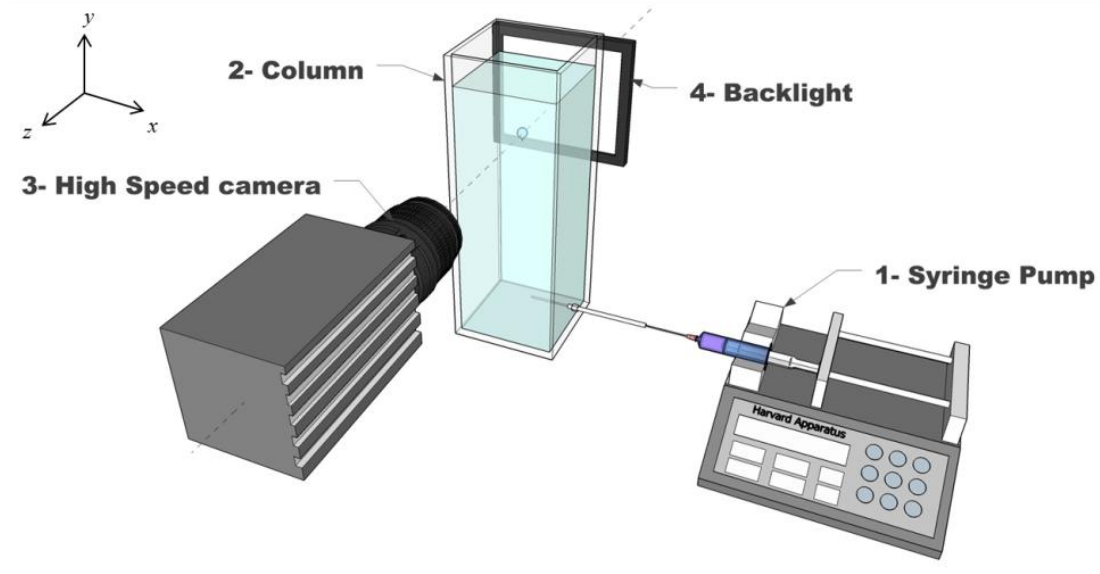

Figure 1. Experimental setup for bubble shape investigation.

\subsection{Materials and the rheological properties}

Two typical non-Newtonian solutions that are widely used in industries [32] were chosen for the experiments: poly(acrylamide-co-acrylic acid) partial sodium salt (PAAm) (Sigma-Aldrich, CAS: 62649-23-4) and Xanthan gum (Sigma-Aldrich, CAS: 11138-66-2). The solutions were prepared by dissolving powder of substances in deionized water, under five concentrations for PAAm $(0.1 \%, 0.25 \%, 0.5 \%, 1 \%, 1.25 \%$ wt.) and three for Xanthan gum $(0.1 \%, 0.25 \%$, $0.5 \%$ wt.). 

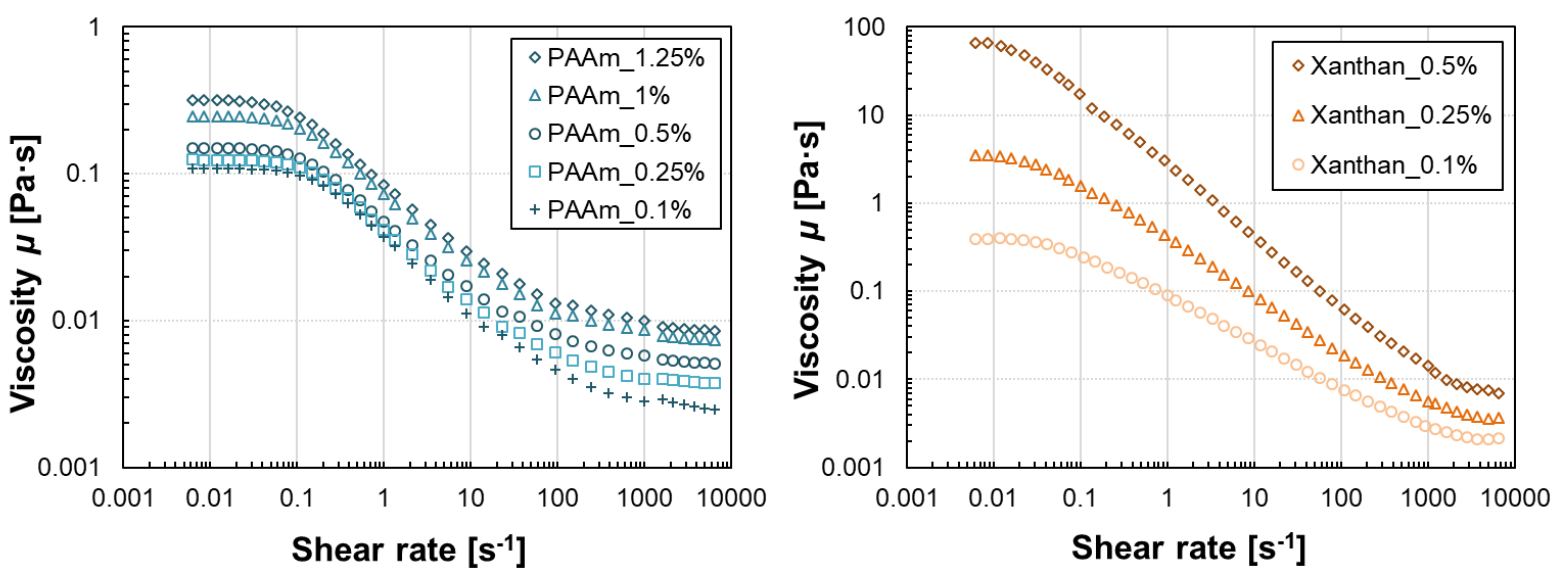

98

Figure 2. Flow curves (viscosity versus shear rate) for PAAm solutions and Xanthan gum solutions

The rheological properties of both PAAm and Xanthan solutions were measured by a rheometer (HAKKE MARS III, Germany) at $293.15 \mathrm{~K}$ with the shear rates ranging from 0.001 $\mathrm{s}^{-1}$ to $10000 \mathrm{~s}^{-1}$. The measured flow curves are plotted in Figure 2. It is found that Xanthan solutions have much higher viscosity than that of the PAAm solutions, especially at low shear rates $\left(<10 \mathrm{~s}^{-1}\right)$. Meanwhile, both of the two solutions present a similar flow behavior. Under low and high shear rates, the viscosity approaches a Newtonian plateau where the value of viscosity trends to be constant. Between these two plateaus, the viscosity decreases with higher shear rate which is known as the shear-thinning property. This evolution can be characterized by the classic Carreau model [33,34]:

$$
\frac{\mu-\mu_{\infty}}{\mu_{0}-\mu_{\infty}}=\left[1+(\lambda \dot{\gamma})^{2}\right]^{\frac{n-1}{2}}
$$

where:

- $\mu$ is the variable viscosity [Pa.s];

- $\mu_{0}$ is the viscosity at the zero shear rate [Pa.s];

- $\mu_{\infty}$ is the viscosity at the infinite shear rate $[\mathrm{Pa} \cdot \mathrm{s}]$;

- $\quad \dot{\gamma}$ is the variable shear rate $\left[\mathrm{s}^{-1}\right]$;

- $\lambda$ is the relaxation time [s];

- $n$ is the power index.

For each solution under different concentrations, multiple samples were tested and fitted with the Carreau model. The fitting results are agreement with the experimental ones with the determination coefficient $r^{2}>97 \%$, proving that the Carreau model is suitable for describing the viscosity variation of the investigated non-Newtonian fluids in our cases. The averaged results of the regression of the viscosity are shown in Table 1 as well as other general physical properties. In this study, the shear rate $\dot{\gamma}$ near the bubble was estimated by $\dot{\gamma}=$ $U / D_{e q}$ with the experimental data: bubble rising velocity $U$ and equivalent diameter $D_{e q}$. This 
126 is the characteristic shear rate near the equator of a spherical bubble. The effective shear

127 rate may be slightly different for large, non-spherical bubbles, but for simplicity we use the

128 same definition in all cases [35]. The methods for calculating $U$ and $D_{e q}$ are presented in

129 Section 2.3 and Section 2.4.1.

130

131 Table 1. Materials and their physical properties ( $T=293.15 \mathrm{~K}$; the viscosity is valid for the 132 shear rate ranging between $0.001 \mathrm{~s}^{-1}$ and $\left.1000 \mathrm{~s}^{-1}\right)$.

\begin{tabular}{cccccccc}
\hline Composition & $\begin{array}{c}\text { Concentration } \\
{[\mathbf{w t}]}\end{array}$ & $\begin{array}{c}\boldsymbol{\sigma} \\
{[\mathrm{mN} / \mathrm{m}]}\end{array}$ & $\begin{array}{c}\rho \\
{\left[\mathbf{k g} / \mathbf{m}^{3}\right]}\end{array}$ & $\begin{array}{c}\boldsymbol{\mu}_{\mathbf{0}} \\
{[\mathrm{Pa} \cdot \mathbf{s}]}\end{array}$ & $\begin{array}{c}\boldsymbol{\mu}_{\infty} \\
{[\mathrm{Pa} \cdot \mathbf{s}]}\end{array}$ & $\begin{array}{c}\lambda \\
{[\mathbf{s}]}\end{array}$ & $\boldsymbol{n}$ \\
\hline PAAM & $0.1 \%$ & 69 & 998 & 0.109 & 0.003 & 5.91 & 0.38 \\
& $0.25 \%$ & 67 & 999 & 0.115 & 0.004 & 6.77 & 0.40 \\
& $0.5 \%$ & 66 & 1000 & 0.120 & 0.005 & 8.19 & 0.42 \\
& $1 \%$ & 65 & 1003 & 0.146 & 0.007 & 9.18 & 0.42 \\
& $1.25 \%$ & 65 & 1004 & 0.159 & 0.008 & 11.86 & 0.43 \\
\hline Xanthan & $0.1 \%$ & 69 & 998 & 0.36 & 0.001 & 15.12 & 0.47 \\
& $0.25 \%$ & 67 & 999 & 3.6 & 0.002 & 26.11 & 0.33 \\
& $0.5 \%$ & 66 & 1001 & 69 & 0.004 & 59.26 & 0.20 \\
\hline
\end{tabular}

134

\subsection{Image processing}

135 The raw images recorded from the experiment cannot be used directly for the bubble shape

136 optimization. An image processing procedure was implemented in MATLAB (R2017b) to

137 extract the bubble profile from the raw images. The specific steps are displayed in Figure 3.

138 Firstly, a reference image concerning the background, which was taken before the passage 139 of the bubble, was subtracted from the raw image (Figure 3-I). The image thus obtained was 140 then transformed into a binary image (Figure $3-\mathrm{II}$ ) on which the bubble was depicted by a 141 black spot after the background noise being removed. Since the bubble may slants while 142 rising in the liquid, a rotation was implemented to situate the major axis of the bubble along 143 the vertical direction. The bubble profile (Figure 3-IV) was finally extracted by recognizing 144 the edge of the bubble spot (Figure 3-III). 


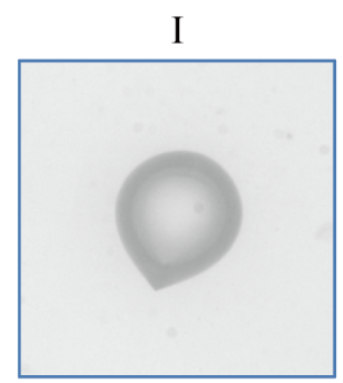

Raw image

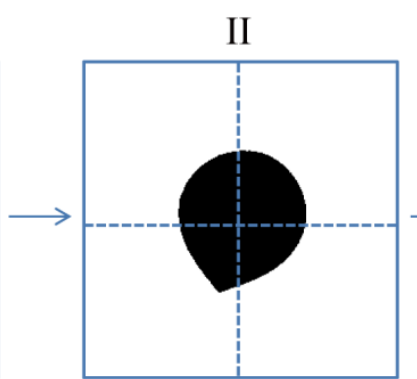

Binary image

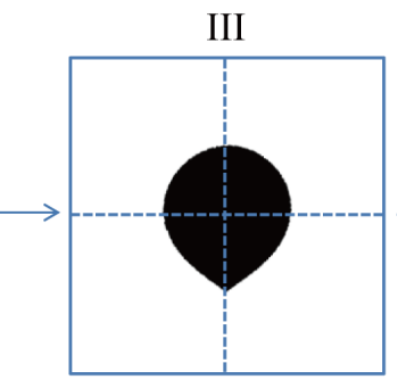

Horizontal image

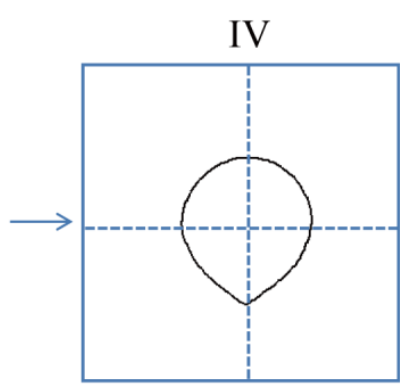

Profile of bubble

Figure 3. Schematic views of the image processing steps (Example of a bubble rising in $0.5 \%$ wt. Xanthan solution).

To calculate the velocity of the bubble, the centroid $\left(x_{i}, y_{i}\right)$ of the bubble spot in each frame of image (Figure 3-II) was recognized. Then the distance between the centroids in two successive frames was divided by the time interval $\Delta t(1 / 2000 \mathrm{~s})$, thus giving the rising velocity of the bubble:

$$
U=\frac{\sqrt{\left(x_{i+1}-x_{i}\right)^{2}+\left(y_{i+1}-y_{i}\right)^{2}}}{\Delta t}
$$

For a given experimental condition, the averaged values of $U$ were calculated by considering 20 pairs of images, and the standard deviation is smaller than $1.25 \%$. Herein it can be assumed that the velocity on the image area has attained the terminal velocity.

After the image processing, the bubble profile is put in the Cartesian coordinates making the bottom of the bubble coincide with the origin and the major axis of the bubble along the ordinate (see Figure 4-I). The coordinates of each point of the bubble profile were then detected to fit the parametric equations which will be discussed in next section.

\subsection{Characterization of bubble shapes}

\subsubsection{Equivalent diameter of the bubble}

As shown in Figure 4-I, the images obtained from the experiment were two-dimensional bubble profiles. To calculate the equivalent diameter of the bubble, a reconstruction of the three-dimensional bubble was implemented considering that the bubble shape was axisymmetric with respect to the major axis of the bubble profile. Then the 3D bubble was divided into a set of multiple small circular truncated cones (Figure 4-II). The volume of each cone equals [36]:

$$
V_{i}=\frac{\pi h}{3}\left(R^{2}+r^{2}+R \cdot r\right)
$$


where $R$ and $r$ are the radius of the lower and upper cross-sections, respectively, and $h$ the height of the cone. These three variables could be directly obtained from the bubble profiles (depicted in Figure 4-I). The total volume of the bubble is the sum of the all the small cones and the equivalent diameter could be calculated as below:

$$
D_{e q}=\sqrt[3]{\frac{6 \sum_{i=1}^{N} V_{i}}{\pi}}
$$
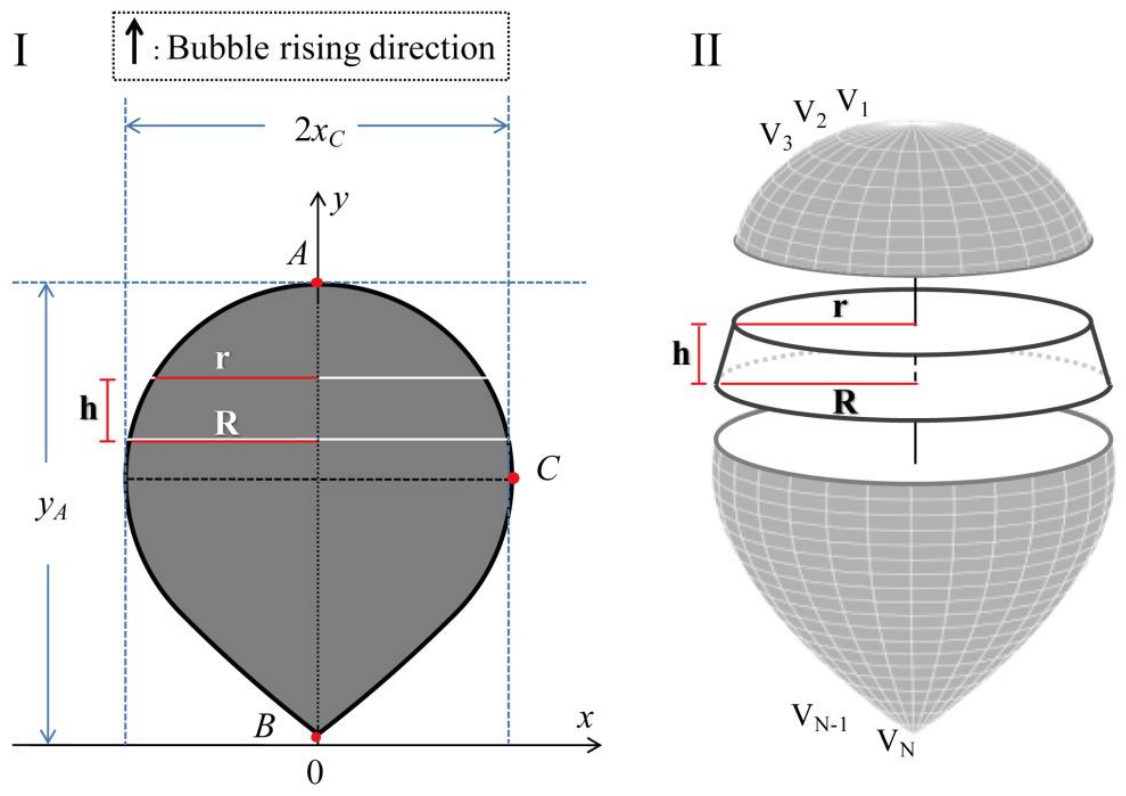

Figure 4. Schematic diagram of the bubble profile (I) and the processing method for the equivalent diameter of the bubble (II).

\subsubsection{Bubble shape equation}

As the example shown in Figure 4, one of the typical shapes in non-Newtonian elastic fluid is the teardrop-like shape. It would be interesting if this kind of shape could be described by simple parametric equations. On the geometry, there exists several mathematical curves which are similar to the teardrop-like bubble shape, such as teardrop, simple folium and piriform. Their parametric equations are given with corresponding profiles in Table 2.

Table 2. Approximate models of the profiles of a bubble or droplet: parametric equation and example.

\begin{tabular}{c|l|c}
\hline Teardrop & $\left\{\begin{array}{l}x=a \sin t\left(\sin \frac{t}{2}\right)^{n} \\
y=a \cos t\end{array}\right.$ \\
& $\begin{array}{c}a=1 \\
n=3 \\
t \in[-\infty,+\infty]\end{array}$ \\
\hline
\end{tabular}




$\left.\begin{array}{l|c|c}\hline \begin{array}{l}\text { Simple } \\ \text { folium }\end{array} & \left\{\begin{array}{l}x=\frac{a t}{1+t^{2^{2}}} \\ y=\frac{a}{1+t^{2}}\end{array}\right. & t \in[-\infty,+\infty]\end{array}\right\}$

189

190 It should be noticed that the equations in the table can only be used to characterize the specific shapes displayed in the right-hand side of table (as shown below), i.e. the shapes

\section{2}

193

194

195

196

197

198 with cusp at the bottom. In order to extend these equations to be used for more types of shapes like spherical, ellipsoidal and cap bubbles, a modification was implemented on the expression of the parametric equation for folium shape by adding extra variables to the euqation. The appropriate expression of the parametric equation was determined through trial and error. A new parametric model is proposed as below:

$$
\left\{\begin{array}{l}
x(t)=\frac{2 x_{C} t}{1+t^{2}} \\
y(t)=\frac{y_{A}}{\left[1+\alpha t^{2}\right]^{\beta}}
\end{array} \quad t \in[-\infty,+\infty]\right.
$$

where:

- $\alpha, \beta$ are two parameters describing the bubble shape;

- $E$ is the aspect ratio;

- $2 x_{C}$ is the length of the minor axis of the bubble profile depicted in Figure 4-I [m];

- $y_{A}$ is the length of the major axis of the bubble profile depicted in Figure 4-I [m];

- $t$ is a numerical variable range between negative and positive infinity.

By adjusting $\alpha$ and $\beta$, the modified parametric equation can be used to describe the profile of different bubble shapes that encountered in non-Newtonian fluids. The validation of the model is discussed in Section 3.1.

In a measurement, the lengths of major axis $y_{A}$ and minor axis $2 x_{C}$ can be obtained directly from the bubble images (Figure 3-III). On the other hand, an optimization should be performed to determine the values of $\alpha$ and $\beta$. Supposing that the edge of the bubble profile can be divided into $N$ points, the coordinates of point $i\left(x_{i, \exp }, y_{i, \exp }\right)$ on the experimental 
212 image are definite. For a pair of arbitrary values $(\alpha, \beta)$, from the function of ordinate in Eq. (5), 213 it has:

$$
t_{i}=\sqrt{\frac{1}{\alpha}\left[\left(y_{i, \exp } / y_{A}\right)^{-\frac{1}{\beta}}-1\right]}
$$

215 By substituting $t_{i}$ back to the function of abscissa in Eq. (5), the calculated abscissa $x_{i, c a l}$ 216 can be obtained as:

$$
x_{i, c a l}=\frac{2 x_{c} t_{i}}{1+t_{i}^{2}}
$$

218 The values of the parameters $\alpha$ and $\beta$ are then optimized by minimizing the sum of the difference at all points between the abscissa calculated with Eq. (8) and the one from the experiment:

$$
\sum_{i=1}^{N}\left|x_{i, c a l}-x_{i, \exp }\right|
$$

The difference is then compared to the equivalent diameter and the relative deviation of the 223 fitting result is defined as follow:

$$
\operatorname{err}=\frac{\sum_{i=1}^{N}\left|x_{i, c a l}-x_{i, e x p}\right|}{N \cdot D_{e q}}
$$

\section{$3 \quad$ Results and discussion}

\section{$227 \quad 3.1$ Validation of the parametric model}

228 Firstly, before dealing with the result from the experiment, the proposed parametric model is 229 verified with respect to its capability for characterizing the bubble shape. According to Eq. (5)

$230 \&(6)$, the parametric equations for the bubble shape involve three parameters: $\alpha, \beta$, and the 231 aspect ratio, $E$. The evolution of the bubble shape in function of $\alpha$ and $\beta$ is depicted in Figure 2325 in which the profiles of the bubbles are shown for aspect ratio $E=1$ (Figure 5-I) and $E=$ 2330.5 (Figure 5-II). It could be observed that the value $E$ controls the global shape that the 234 profile becomes more oblate with smaller value $E$ (e.g. spherical bubble to ellipsoidal bubble). 235 On the other hand, the parameters $\alpha$ and $\beta$ control the detail of the shape. The value of $\alpha$ 236 can change the position of the minor axis (horizontal) of the bubble profiles. When the value 237 of $\beta$ is fixed (e.g. $\beta=1$ ) and $\alpha$ increases from 0.5 to 2.5 , the minor axis is moving from near 238 the top of the bubble to the bottom of the bubble. On the other hand, $\beta$ is related to the 239 appearance of the cusp at the bottom of the bubble. Herein, the bubble deformation can be 240 roughly summarized as follows: 
- For $\beta<0.6$, there is a clear cusp at the bottom of the bubble. The typical inverted teardrop shape appears at a high value of $\alpha$. (e.g. Figure 5-I: $(\alpha, \beta)=(2.5,0.4)$ ).

- For $0.6<\beta<0.8$, the bubble becomes progressively rounded at the bottom and the cusp gradually disappears as the value of $\beta$ increases.

- For $0.8<\beta<1$, there is no longer a cusp and the profile becomes more and more rounded. The quasi-ellipsoidal bubble appears at this interval. In particular, the circular profile (spherical bubble) and the perfectly elliptical profile (ellipsoidal bubble) appear when the value $\alpha$ and $\beta$ are both equal to 1 .

- For $\beta>1$, the bottom of the profile tends to be flat. The typical cap shape appears when the values of $\alpha$ and $\beta$ are both sufficient large (e.g. Figure 5 -II: $\alpha=1.5, \beta=1.5$ ).
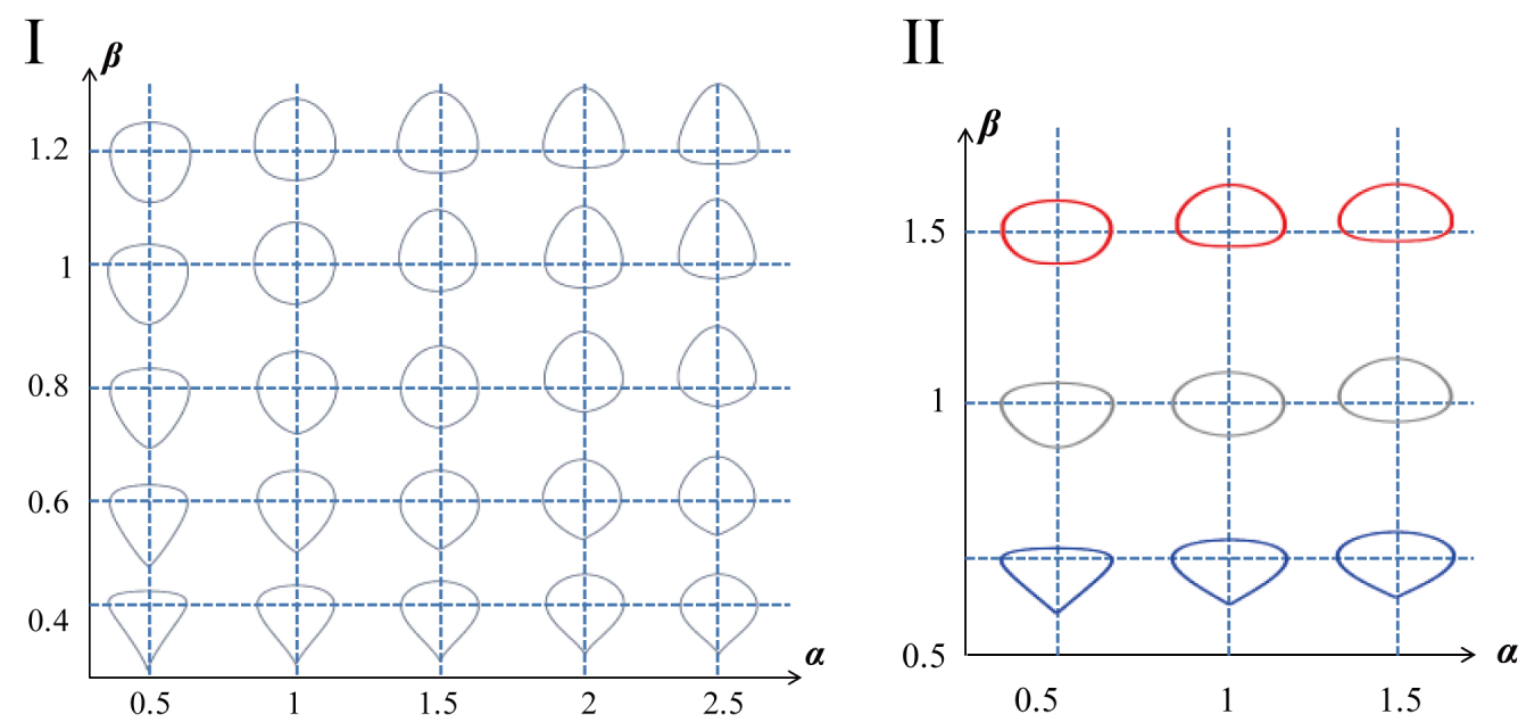

Figure 5. Evolution of the shape versus the parameters $\alpha$ and $\beta(\mathrm{I}: E=1, \mathrm{II}: E=0.5)$.

Therefore, by adjusting the values of parameters $\alpha, \beta$ and $E$, the majority of the bubble shapes appeared in non-Newtonian fluids can be characterized precisely. Based on the parametric equations, the investigation of the bubble shape can be carried out by analyzing the evolution of these parameters.

\subsection{Bubble shape regimes}

260 The resulting bubble shapes in different non-Newtonian solutions (i.e. both PAAm and 261 Xanthan gum solutions) are shown as bubble shape regime map, namely so-called Grace 262 Map, in Figure 6. This bubble shape regime map is plotted in terms of the dimensionless $R e$, 263 Eo numbers and log Mo (dark gray curves), which can be calculated as defined in Eq.(11)- 
(13), and the typical bubble shapes (gray patterns in the background) extracted from the raw images (Figure 3-III) are also depicted near the corresponding experimental points.

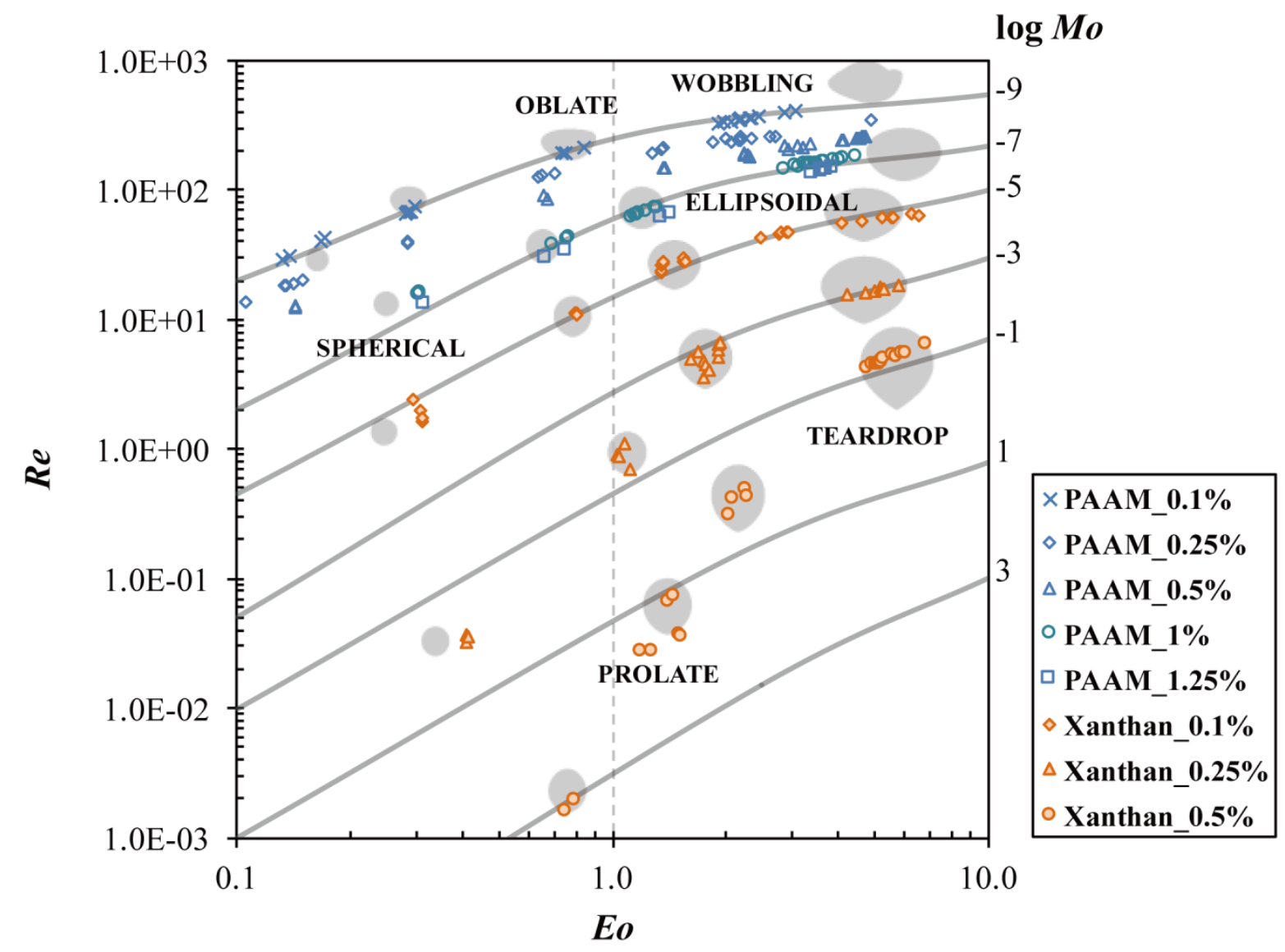

Figure 6. Shape regime map (namely Grace Map) for bubbles in PAAm and Xanthan gum solutions (dark gray curves: log Mo; gray patterns in the background: typical bubble shape near the corresponding experimental points).

$$
M o=\frac{g \mu^{4}}{\rho \sigma^{3}}
$$

274 In PAAm solutions, the range of Mo number is relatively small $(-9<\log \mathrm{Mo}<-7)$ within the 275 concentration range of study $(0.1 \%-1.25 \%$ wt.). The data covers the spherical bubble and 276 the ellipsoidal bubble regimes. When increasing Eo and $R e$, the bubble becomes bigger and 277 the shape changes from spherical to ellipsoidal. Regarding the concentration of the solution, 278 it can be seen that the $R e$ increases in a dilute solution, making the bubble shape become 279 more oblate and even causing wobbling due to the surface oscillation. 
In Xanthan solutions, the range of $M o$ number is larger $(-9<\log \mathrm{Mo}<3)$ under the investigated concentration $(0.1 \%-0.5 \%$ wt.). The data covers not only the spherical and ellipsoidal bubbles but also the prolate and teardrop ones. A similar tendency is found for the bubble deformation as in the PAAm solutions. In particular, the cusp appears for high Eo number $(E o>1)$ and becomes more significant with the increase of the solution concentration or for lower Re number. Under this condition, the bubble is also elongated vertically, which is uncommon in Newtonian fluids.

287 Compared with the Grace map given by Clift et al. [37] for bubble shapes in Newtonian fluids, 288 the regimes of the spherical bubble and the ellipsoidal bubble in the present study are 289 consistent when $R e>1$. However, the bubble shapes are invariably spherical when $R e<1$, which are contrary to those in non-Newtonian fluids. In fact, in non-Newtonian fluids, as the bubble velocity could be extremely low due to the complex rheological properties of the solution, relatively smaller values of $R e$ number should be considered, namely $R e$ ranging from 0.001 to 1 in this study. Within this range, the prolate bubble and teardrop bubble can also be visualized while the spherical bubble appears only for low Eo number $(E 0<1)$.

\subsection{Bubble velocity}

297 Concerning the bubble terminal velocity, as shown in Figure 7, for both types of the solutions, 298 it first increases with the bubble size then tends to be constant. Although the velocity curves at different concentrations differ more from each other for Xanthan solutions, this evolution and the final constant velocity do not depend on the type of solution or the concentration of the solute. It could be derived that, in a highly viscous Xanthan solution, the bubble rises much more slowly and needs to attain the final constant velocity in a bigger size. In particular, it exists a remarkable difference between the curves of the three lower concentrations of PAAm solutions and those of the two higher concentrations. The reason is that, in the dilute PAAm solutions $(0.1 \%-0.5 \% \mathrm{wt})$, the bubble shape is less stable with the surface oscillation, which leads to the reduction of the viscosity around the bubble due to the shear thinning property. On the other hand, when a bubble is rising in a concentrated PAAm solution (1\%$1.25 \% \mathrm{wt}$ ), the surface oscillation no longer exists due to the higher viscosity. The bubble thus encounters more resistance and its velocity is much slower. Moreover, there is no evident velocity discontinuity under our experimental conditions indicating that the bubble terminal velocity could be less influenced by the bubble shape deformation (from ellipsoidal

312 to inverted teardrop). This result is consistent with the literature [38], in which the same 313 behavior of rising bubbles was found in CMC solutions and Xanthan gum solutions. 

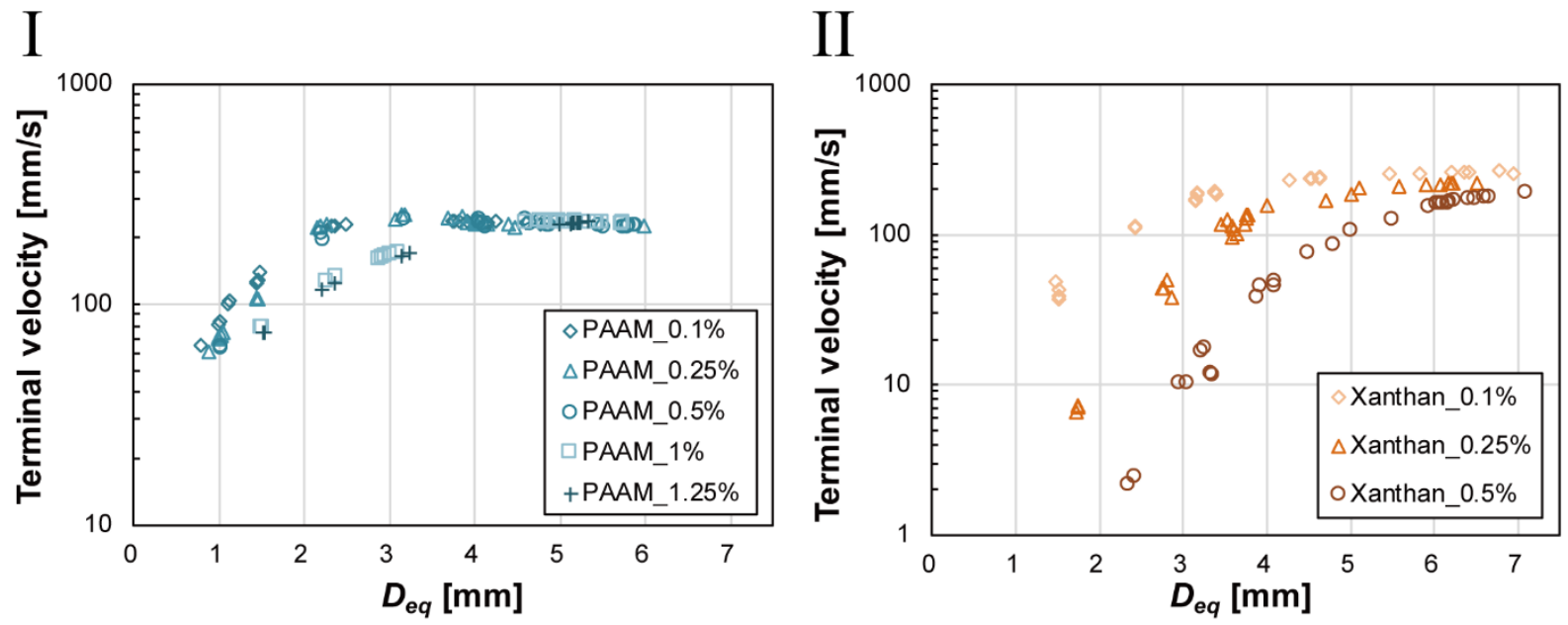

Figure 7. Relationship between bubble rising velocity and bubble size $\left(D_{e q}=0.7 \sim 7 \mathrm{~mm}\right)$ in PAAm (I) and Xanthan gum solutions (II).

\subsection{Bubble shape parameters}

319 To better analyze the bubble shape, the bubble profile was fitted by the parametric models introduced in Section 2.4.2. It should be noticed that the bubble shape is assumed to be stable without surface oscillation. The data for big bubble $\left(D_{e q}>6 \mathrm{~mm}\right)$ rising in dilute PAAm solution $(0.1 \%-0.5 \% \mathrm{wt})$ has been eliminated due to the non-negligible surface oscillation. Two typical fitting results, chosen for single bubbles in Xanthan gum and PAAm solutions, are shown in Figure 8-I \& II, where the black dots depict the origin profile of the bubble and the red line is the fitting profile with the values of $\alpha$ and $\beta$ given alongside. It can be seen that whether the bubble has a teardrop or an ellipsoidal shape, the deviation between the experimental points and the fitting points is small. The deviation defined in Eq. (10) is in a magnitude smaller than $3 \%$ for all the cases. To ensure the validity of the bubble characterization result, fitting data with an error of more than $2 \%$ was eliminated. An example of the averaged fitting results of the bubble shapes in both PAAm and Xanthan solutions under different concentrations are shown in Figure 8-III \& IV. It can be observed that under the same operational condition for bubble generation (i.e. the size of nozzle and the air flow rate), the sizes and shapes of bubbles depend on the type and concentration of the solutes (i.e. PAAm and Xanthan gum). In addition, the evolution of the bubble shapes given by the model is consistent with the original bubble shapes depicted in Figure 6. 
I

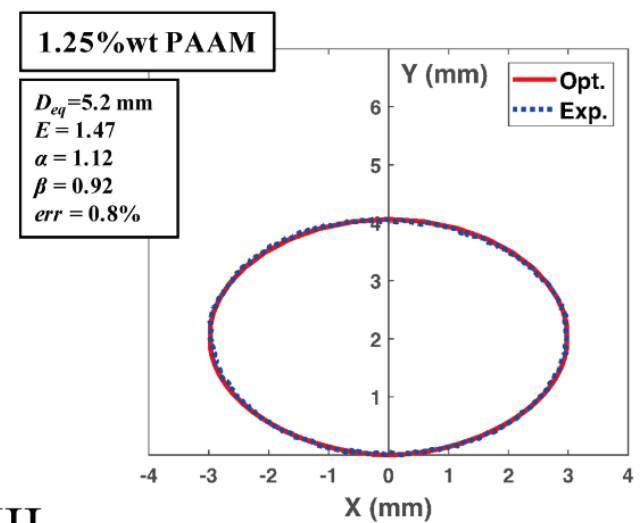

III

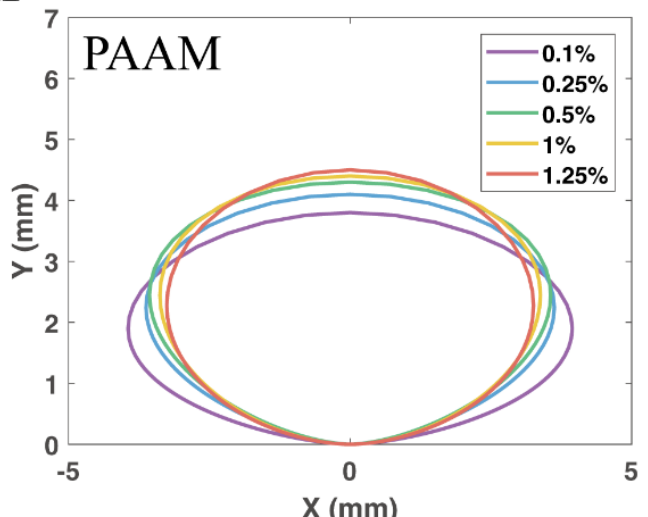

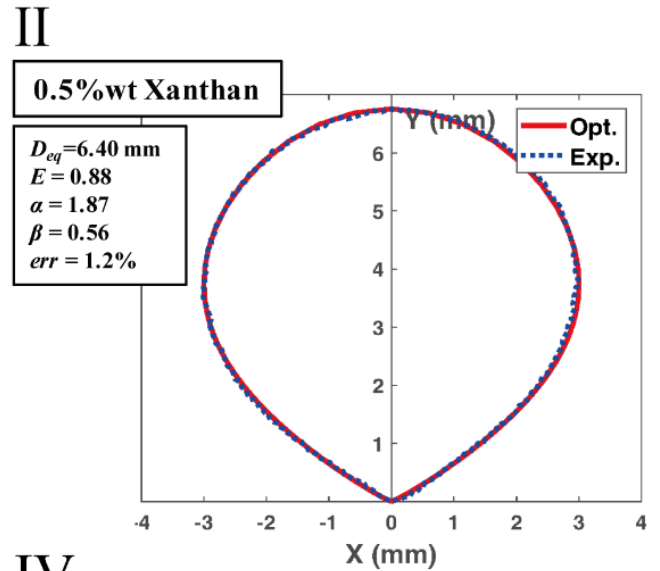

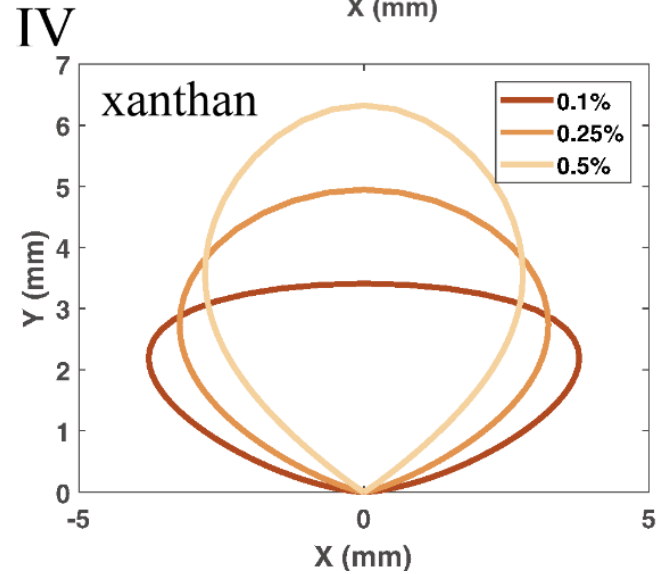

Figure 8. Examples of the bubble profiles and the fitting ones by Eq. (5) \& (6) in PAAm (I, III) and Xanthan solutions (II, IV) under different concentrations. (The bubbles are injected by the nozzle with a caliber of $1 \mathrm{~mm}$ at a flow rate of $10 \mu \mathrm{L} / \mathrm{h}$ ).

It can be seen from Figure 8-III \& IV that in PAAm solution, the size of the bubble slightly changes, while the shape becomes more and more rounded for higher concentrations, namely, from an oblate bubble to a general ellipsoidal bubble. In contrast, in Xanthan solutions, the major axis (vertical) of the bubble increases as the concentration of Xanthan increases, as well as the bubble deforms from an oblate ellipsoid to an oblate teardrop and then turns to an alongate teardrop.

For a single bubble, a pair of parameters $(\alpha, \beta)$ could be obtained from the fitting result. In Figure 9, the relationship between the bubble shape parameters $(\alpha$ and $\beta$ ) are depicted for bubbles in both PAAm and Xanthan gum solutions. The corresponding bubble profiles (light gray outline) are also displayed at the positions of values $\alpha$ and $\beta$. In this figure, the delicate changes of bubble shape can be visualized and quantified, which is difficult to achieve simply by using the aspect ratio. For PAAm solutions, the data are concentrated in the range of $1<\alpha$ $<1.2$ and $0.8<\beta<1$, suggesting that the bubble is spherical or ellipsoidal, while, for the Xanthan solution, the range of these two parameters is much larger. As the parameter $\alpha$ increases, the value of $\beta$ decreases gradually. As interpreted in Section 2.4.2, the cusp of 
356 the bubble appears when $\beta<0.6$ and it becomes sharper when $\beta$ continues to decrease.

357 The result of the Xanthan gum cases shows good agreement with this evolution.

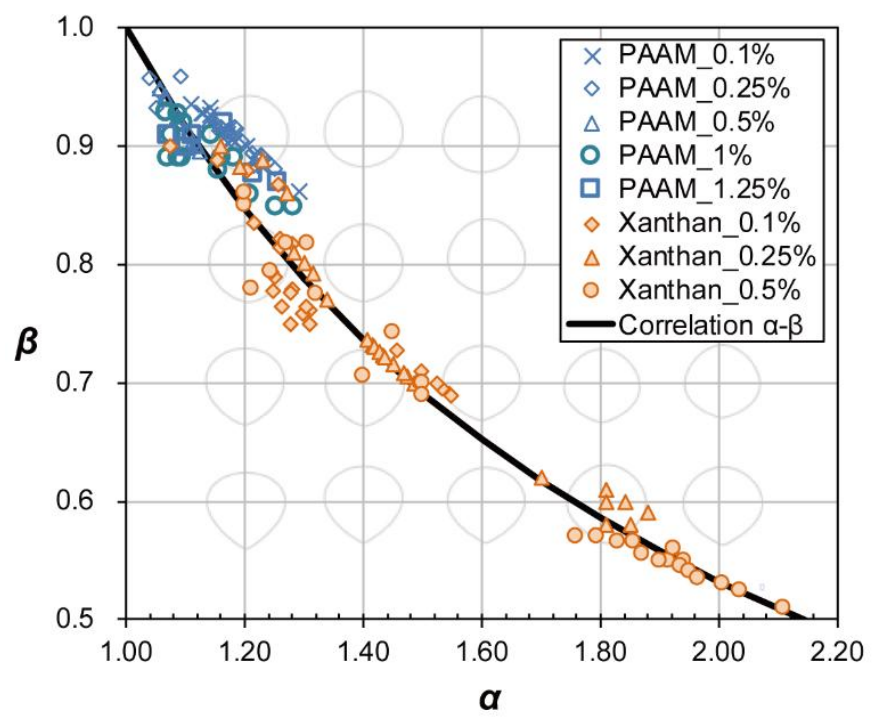

Figure 9. Relationship between the bubble shape parameters ( $\alpha$ and $\beta$ ) in both PAAm and Xanthan solutions under different concentrations.

It is found that the relationship between $\alpha$ and $\beta$ could be correlated by a power-law function, which is expressed as below:

$$
\alpha^{0.85} \beta=1
$$

In the range investigated in this study $(-9<\log M o<3)$, the accuracy of this correlation is acceptable, with a determination coefficient $r^{2}>95 \%$. It can be seen from Figure 9 that the curve of Eq. (14) passes the point $(\alpha, \beta)=(1,1)$, which indicates a perfect spherical bubble (or an ellipsoidal bubble when $E \neq 1$ ). Thanks to Eq. (14), it is possible to characterize the bubble shape with only one parameter (either $\alpha$ or $\beta$ ). In the next section, only the parameter $\beta$ is taken into account.

\subsection{Prediction of bubble shape}

373 It is known from Section 3.1 that the aspect ratio $E$ roughly describes the bubble shape while

374 the parameter $\alpha$ and $\beta$ characterize the details of bubble deformation. As mentioned in the 375 introduction that a lot of research has been carried out for predicting the aspect ratio $E$ for 376 bubbles in different liquids, we will focus on the discussion of the bubble shape parameter $\beta$.

377 Depending on the value of $\beta$, bubble shape changes from ellipsoidal (without cusp) to 378 teardrop (with cusp). Some existed research attempts to explain the appearance of the cusp 
379 theoretically and most of the authors support the reason that the majority of non-Newtonian

380 fluids are composed of long-chain molecules and possess viscoelasticity [39,40]. To quantify 381 the elasticity of the fluid, the Deborah number is utilized to compare the relaxation time of the 382 fluid to the time scale of observation [41]:

$$
D e=\frac{\lambda U}{D_{e q}}=\lambda \dot{\gamma}
$$

384 In our study, the observation time is estimated that it is equal to the characteristic time of the motion (i.e. the equivalent diameter of the bubble divided by the bubble rising velocity). Since 386 this characteristic time of the motion is relatively short comparing with the relaxation time of 387 the fluid, the magnitude of calculated De number in this study may be higher than the 388 literature [42]. Nonetheless, it can still be used to compare the elastic levels for the 389 investigated fluids.

390 Since the cusp could now be quantified by the parameter $\beta$, the evolution of $\beta$ is analyzed in terms of the bubble size $(V)$ and various dimensionless numbers $(D e, E o, R e)$, shown as in Figure 10.

I

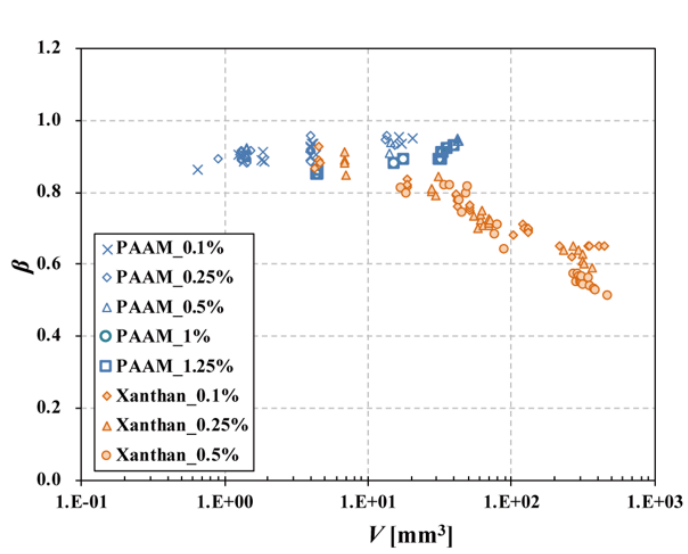

\section{III}

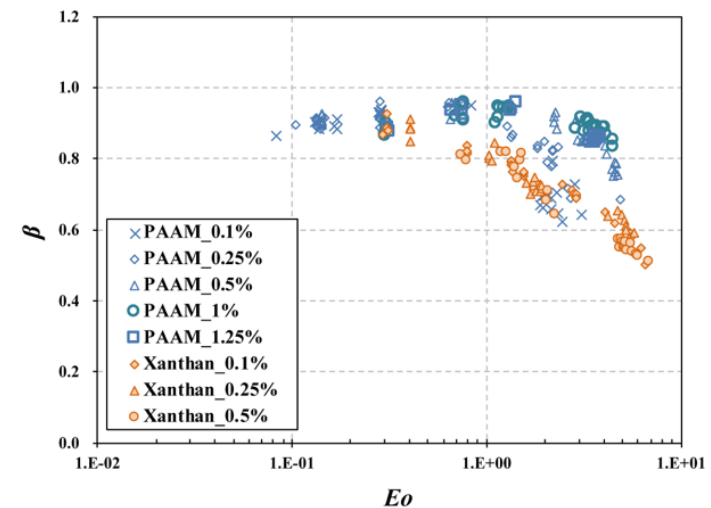

II

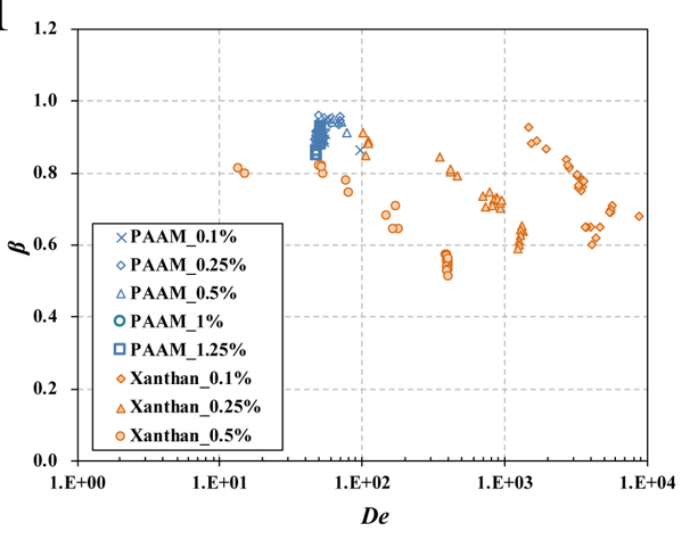

IV

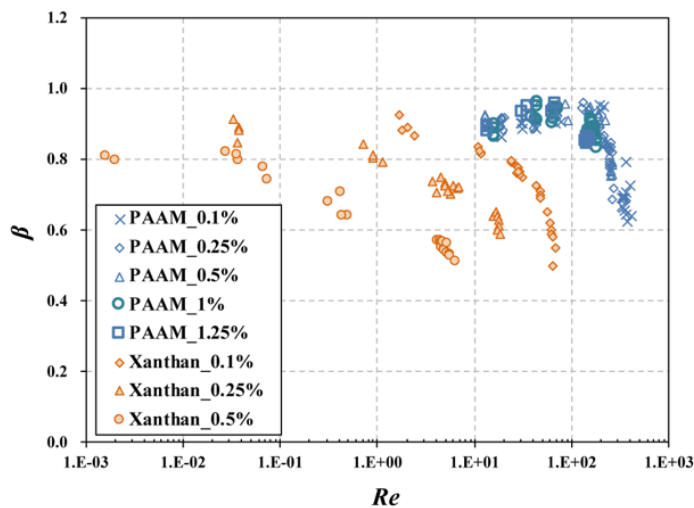


Figure 10. The evolution of parameter $\beta$ in terms of the bubble size $(V)$ and various dimensionless numbers ( $D e, E o, R e)$ in PAAm and Xanthan solutions under different concentrations.

It can be obtained from Figure 10 that: (1) for larger bubbles, the velocity of the bubble increases then the bubble begins to deform and becomes less rounded when the contribution of inertial force and buoyancy is stronger than the surface tension and viscous force. (2) $\beta$ decreases with higher elasticity of the solution in most investigated range except 403 for some PAAm which are relatively less elastic. If the bubble moves too fast, the liquid 404 cannot fill the space in the rear of the bubble immediately. Hence this space is still occupied by the gas and pressed by the long-chain liquid molecule from the side, leading to a little cusp forming in the rear of the bubble. (3) In the evolution of $\beta-D e$ and $\beta-R e$, the evolution of the value $\beta$ under three concentrations is quite dispersed. This result might be caused by the different shear-thinning level of the solutions as the shear-thinning property is also a reason for the deformation of the bubble [43].

410 The data in Figure $\mathbf{1 0}$ are too scattered for a general law to be obtained. Hence multiple 411 influencing factors are considered instead of one. By analogy with the analysis of aspect ratio, 412 the investigation was carried out for the evolution of $\beta$ with different pairs of dimensionless 413 numbers as well as the shear-thinning property which can be roughly quantified by the power 414 index $n$ (Eq. (1)). After testing several functions, the following correlation was proposed to fit 415 the experimental data:

$$
\beta=n-0.11 D e^{0.19} E o^{0.11}+0.80
$$

417 This equation is correlated to the result depicted in Figure $\mathbf{1 0}$ as $\beta$ decreases monotonically 418 with high $D e$, Eo numbers and the shear-thinning levels. The comparison between parameter $419 \beta$ predicted by Eq. (16) and the experimental ones is shown in Figure 11. It can be seen that, 420 under the range of this study $(-9<\log M o<3)$, the prediction quality of parameter $\beta$ in both 421 PAAm and Xanthan gum solutions is satisfactory. Relatively large deviation appears at high 422 values of $\beta$ due to the wobbling of the bubble rising in dilute PAAm solutions. The fitting 423 result has a coefficient of determination $\left(r^{2}\right)$ higher than $95 \%$. 


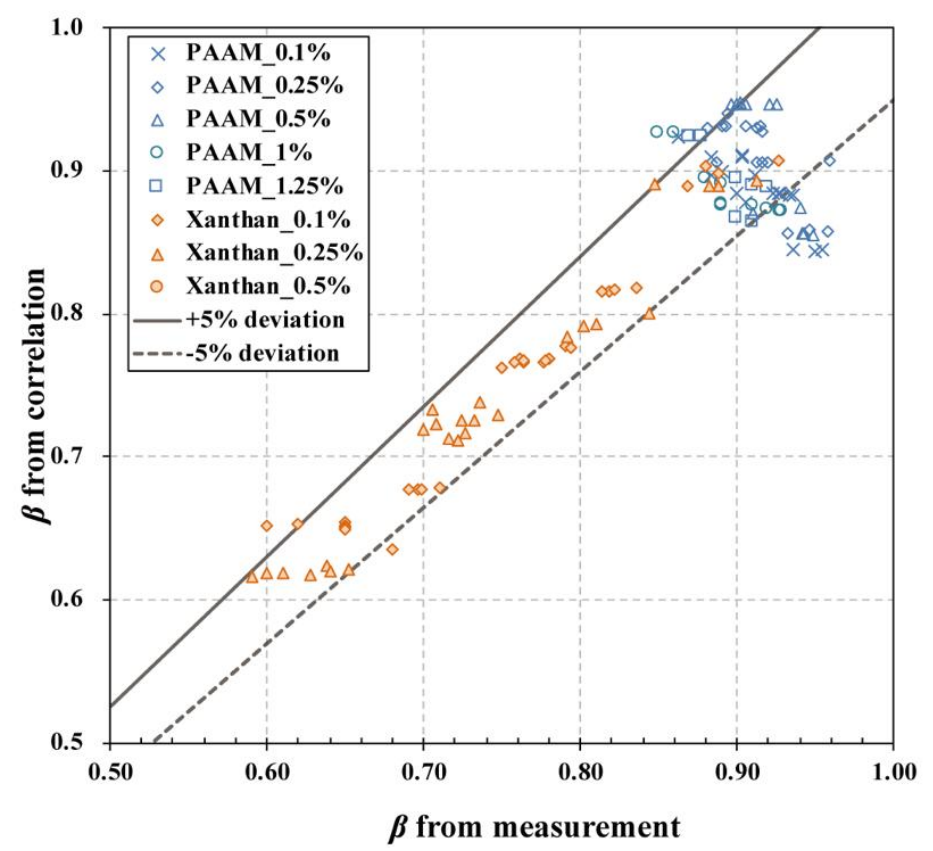

424

425 Figure 11. Comparison result between parameter $\beta$ from correlation (Eq .(16)) and $\beta$ from 426 measurement in PAAM and Xanthan gum solutions.

\section{$4 \quad$ Conclusions}

429 In this paper, various single air bubbles rising in stagnant non-Newtonian fluids 430 (polyacrylamide, Xanthan gum) were investigated by high-speed photography. The shapes of 431 the bubbles from spherical to inverted teardrop can be characterized with good accuracy by 432 parametric equations which contain the aspect ratio $(E)$ and two shape parameters $(\alpha, \beta)$. 433 Based on this method, the various impacts on the bubble shape can be quantified by 434 studying the evolution of the three parameters as a function of bubble size, bubble velocity, 435 multiple dimensionless numbers ( $R e, E o$, etc.) and the property of the fluids. Within the range 436 investigated here $(-9<\log \mathrm{Mo}<-7)$, it is found that the cusp of the bubble (quantified by $\beta)$ is 437 related to the viscoelasticity as well as the shear-thing level of the solutions. A simple 438 correlation is then proposed to predict the parameter $\beta$. Since the parametric equations are 439 capable to characterize most of observed shapes (including cap bubble), this method can be 440 applied to bigger bubble or droplets in complex Newtonian and non-Newtonian fluids. 


$\begin{array}{lll}D_{e q} & {[\mathrm{~mm}]} & \text { equivalent diameter } \\ E & {[-]} & \text { aspect ratio } \\ E o & {[-]} & \text { Eötvös number } \\ g & {\left[\mathrm{~m} \cdot \mathrm{s}^{-2}\right]} & \text { gravitational acceleration } \\ \text { Mo } & {[-]} & \text { Morton number } \\ n & {[-]} & \text { power index / shear-thing level } \\ P & {[\mathrm{~mm}]} & \text { perimeter } \\ r & {[-]} & \text { determination coefficient } \\ R e & {[-]} & \text { Reynolds number } \\ S & {\left[\mathrm{~mm}^{2}\right]} & \text { surface area } \\ t & {[-]} & \text { numerical variable } \\ T a & {[-]} & \text { Tadaki number } \\ U_{x} & {\left[\mathrm{~mm} \cdot \mathrm{s}^{-1}\right]} & \text { oscillating velocity } \\ U_{y} & {\left[\mathrm{~mm} \cdot \mathrm{s}^{-1}\right]} & \text { rising velocity } \\ V & {\left[\mathrm{~mm}{ }^{3}\right]} & \text { Bubble volume } \\ W e & {[-]} & \text { Weber number } \\ x_{i, \text { cal }} & {[\mathrm{mm}]} & \text { abscissa by calculation } \\ x_{i, \text { exp }} & {[\mathrm{mm}]} & \text { abscissa by experiment } \\ x_{C} & {[\mathrm{~mm}]} & \text { half-length of major axis } \\ y_{A} & {[\mathrm{~mm}]} & \text { length of minor axis }\end{array}$

\section{Greek Symbols}

$\begin{array}{lll}\alpha & {[-]} & \text { bubble shape parameter } \\ \beta & {[-]} & \text { bubble shape parameter } \\ \dot{\gamma} & {\left[\mathrm{s}^{-1}\right]} & \text { shear rate } \\ \lambda & {[\mathrm{s}]} & \text { relaxation time } \\ \mu & {[\mathrm{Pa} \cdot \mathrm{s}]} & \text { dynamic viscosity } \\ \rho & {\left[\mathrm{kg} \cdot \mathrm{m}^{-3}\right]} & \text { density } \\ \sigma & {\left[\mathrm{N} \cdot \mathrm{m}^{-1}\right]} & \text { surface tension }\end{array}$

\section{Acknowledgments}

445 The financial assistance provided for Feishi. XU by the China Scholarship Council is 446 gratefully acknowledged. The federation FERMAT is also thanked for its leading-edge 447 material support.

\section{References}

450 [1] R.P. Chhabra, Bubbles, Drops, and Particles in Non-Newtonian Fluids, Second 451 Edition, 2 edition, CRC Press, 2006.

452 [2] F. Xu, A. Cockx, G. Hébrard, N. Dietrich, Mass Transfer and Diffusion of a Single 453 Bubble Rising in Polymer Solutions, Ind. Eng. Chem. Res. (2018). 
doi:10.1021/acs.iecr.8b03617.

455 [3] J. Grace, T. Wairegi, T.H. Nguyen, Shapes and Velocities of Single Drops and 456 Bubbles Moving Freely Through Immiscible Liquids, Trans Inst Chem Eng. 54 (1976) 167457173.

458 [4] S. Anwar, Lattice Boltzmann modeling of buoyant rise of single and multiple bubbles, 459 Comput. Fluids. 88 (2013) 430-439. doi:10.1016/j.compfluid.2013.09.015.

460 [5] J. Hua, J.F. Stene, P. Lin, Numerical simulation of 3D bubbles rising in viscous liquids 461 using a front tracking method, J. Comput. Phys. 227 (2008) 3358-3382.

462 doi:10.1016/j.jcp.2007.12.002.

463 [6] Z. Yu, H. Yang, L.-S. Fan, Numerical simulation of bubble interactions using an 464 adaptive lattice Boltzmann method, Chem. Eng. Sci. 66 (2011) 3441-3451.

465 doi:10.1016/j.ces.2011.01.019.

466 [7] L. Böhm, T. Kurita, K. Kimura, M. Kraume, Rising behaviour of single bubbles in 467 narrow rectangular channels in Newtonian and non-Newtonian liquids, Int. J. Multiph. Flow. 46865 (2014) 11-23. doi:10.1016/j.jmultiphaseflow.2014.05.001.

469 [8] X. Frank, H.Z. Li, D. Funfschilling, F. Burdin, Y. Ma, Bubble Motion in Non-Newtonian 470 Fluids and Suspensions, Can. J. Chem. Eng. 81 (2003) 483-490.

471 doi:10.1002/cjce.5450810321.

472 [9] X. Frank, J.-C. Charpentier, Y. Ma, N. Midoux, H.Z. Li, A Multiscale Approach for 473 Modeling Bubbles Rising in Non-Newtonian Fluids, Ind. Eng. Chem. Res. 51 (2012) 2084474 2093. doi:10.1021/ie2006577.

475 [10] N.M.S. Hassan, M.M.K. Khan, D.W. Rackemann, An experimental study of bubble 476 rise characteristics in non-Newtonian (power-law) fluids, 16th Australas. Fluid Mech. Conf. 477 AFMC. (2007). http://acquire.cqu.edu.au:8080/vital/access/manager/Repository/cqu:2807 478 (accessed January 31, 2017).

479 [11] N.M.S. Hassan, M.M.K. Khan, M.G. Rasul, D.W. Rackemann, Bubble Rise Velocity 480 and Trajectory in Xanthan Gum Crystal Suspension, Appl. Rheol. 20 (2010) 65102.

481 [12] L. Zhang, C. Yang, Z.-S. Mao, Numerical simulation of a bubble rising in shear482 thinning fluids, J. Non-Newton. Fluid Mech. 165 (2010) 555-567.

483 doi:10.1016/j.jnnfm.2010.02.012.

484 [13] D. Dekée, R.P. Chhabra, A photographic study of shapes of bubbles and 485 coalescence in non-Newtonian polymer solutions, Rheol. Acta. 27 (1988) 656-660. 486 doi:10.1007/BF01337462. 
[14] D. Dekée, P.J. Carreau, J. Mordarski, Bubble velocity and coalescence in viscoelastic liquids, Chem. Eng. Sci. 41 (1986) 2273-2283. doi:10.1016/0009-2509(86)85078-3.

489 [15] D. Funfschilling, H.Z. Li, Effects of the Injection Period on the Rise Velocity and 490 Shape of a Bubble in a Non-Newtonian Fluid, Chem. Eng. Res. Des. 84 (2006) 875-883. 491 doi:10.1205/cherd.01229.

492 [16] N. Dietrich, G. Hebrard, Visualisation of gas-liquid mass transfer around a rising 493 bubble in a quiescent liquid using an oxygen sensitive dye, Heat Mass Transf. (2018) 1-9. 494 doi:10.1007/s00231-018-2297-3.

495 [17] N. Dietrich, K. Wongwailikhit, M. Mei, F. Xu, F. Felis, A. Kherbeche, G. Hébrard, K. 496 Loubière, Using the "Red Bottle" Experiment for the Visualization and the Fast

497 Characterization of Gas-Liquid Mass Transfer, J. Chem. Educ. (2019).

498 doi:10.1021/acs.jchemed.8b00898.

499 [18] A. Kherbeche, M. Mei, M.-J. Thoraval, G. Hébrard, N. Dietrich, Hydrodynamics and 500 gas-liquid mass transfer around a confined sliding bubble, Chem. Eng. J. (2019).

501 doi:10.1016/j.cej.2019.04.041.

502 [19] D.W. Moore, The rise of a gas bubble in a viscous liquid, J. Fluid Mech. 6 (1959) 503 113-130. doi:10.1017/S0022112059000520.

504 [20] T. Tadaki, S. Maeda, On the Shape and Velocity of Single Air Bubbles Rising in 505 Various Liquids, Chem. Eng. 25 (1961) 254-264. doi:10.1252/kakoronbunshu1953.25.254.

506 [21] T.D. Taylor, A. Acrivos, On the deformation and drag of a falling viscous drop at low 507 Reynolds number, J. Fluid Mech. 18 (1964) 466-476. doi:10.1017/S0022112064000349.

508 [22] R.M. Wellek, A.K. Agrawal, A.H.P. Skelland, Shape of liquid drops moving in liquid 509 media, AIChE J. 12 (1966) 854-862. doi:10.1002/aic.690120506.

510 [23] I.A. Vakhrushev, G.I. Efremov, Interpolation formula for computing the velocities of 511 single gas bubbles in liquids, Chem. Technol. Fuels Oils. 6 (1970) 376-379.

512 doi:10.1007\%2fBF01171684.

513 [24] L.-S. Fan, K. Tsuchiya, Bubble Wake Dynamics in Liquids and Liquid-Solid 514 Suspensions, Butterworth-Heinemann, Boston, Mass, 1990.

515 [25] F. Raymond, J.-M. Rosant, A numerical and experimental study of the terminal 516 velocity and shape of bubbles in viscous liquids, Chem. Eng. Sci. 55 (2000) 943-955. 517 doi:10.1016/S0009-2509(99)00385-1.

518 [26] K. Sugihara, T. Sanada, M. Shirota, M. Watanabe, Behavior of single rising bubbles 519 in superpurified water, KAGAKU KOGAKU RONBUNSHU. 33 (2007) 402-408. 
520 [27] D. Legendre, R. Zenit, J.R. Velezcordero, On the deformation of gas bubbles in 521 liquids, Phys. Fluids. 24 (2012) 043303.

522 [28] G. Kelbaliyev, K. Ceylan, Development of New Empirical Equations for Estimation of 523 Drag Coefficient, Shape Deformation, and Rising Velocity of Gas Bubbles or Liquid Drops, 524 Chem. Eng. Commun. 194 (2007) 1623-1637. doi:10.1080/00986440701446128.

525 [29] L. Liu, H. Yan, G. Zhao, Experimental studies on the shape and motion of air bubbles 526 in viscous liquids, Exp. Therm. Fluid Sci. 62 (2015) 109-121.

527 doi:10.1016/j.expthermflusci.2014.11.018.

528 [30] W. Myint, S. Hosokawa, A. Tomiyama, Shapes of Single Drops Rising Through 529 Stagnant Liquids, J. Fluid Sci. Technol. 2 (2007) 184-195. doi:10.1299/jfst.2.184.

530 [31] H. Yan, J. Zhao, Experimental study on shape and rising behavior of single bubble in 531 stagnant water, J. Cent. South Univ. Sci. Technol. 47 (2016) 2513-2520.

532 [32] R.L. Davidson, Handbook of water-soluble gums and resins, McGraw-Hill, 1980.

533 [33] R. Byron Bird, P.J. Carreau, A nonlinear viscoelastic model for polymer solutions and 534 melts-I, Chem. Eng. Sci. 23 (1968) 427-434. doi:10.1016/0009-2509(68)87018-6.

535 [34] P.J. Carreau, I.F. MacDonald, R.B. Bird, A nonlinear viscoelastic model for polymer 536 solutions and melts-II, Chem. Eng. Sci. 23 (1968) 901-911. doi:10.1016/0009-

537 2509(68)80024-7.

538 [35] N. Dietrich, Étude locale et expérimentale des phénomènes interfaciaux, 539 Vandoeuvre-les-Nancy, INPL, 2008. http://www.theses.fr/2008INPL078N (accessed 540 September 20, 2016).

541 [36] J.W. Harris, H. Stöcker, Handbook of Mathematics and Computational Science, 1998 542 edition, Springer, New York, 1998.

543 [37] R. Clift, J.R. Grace, M.E. Weber, Bubbles, Drops, and Particles, Dover Publications, 544 New York, 1978.

545 [38] S. Amirnia, J.R. de Bruyn, M.A. Bergougnou, A. Margaritis, Continuous rise velocity of 546 air bubbles in non-Newtonian biopolymer solutions, Chem. Eng. Sci. 94 (2013) 60-68.

547 doi:10.1016/j.ces.2013.02.032.

548 [39] S.M. Barnett, A.E. Humphrey, M. Litt, Bubble motion and mass transfer in non549 Newtonian fluids, AIChE J. 12 (1966) 253-259.

550 [40] R. Zenit, J.J. Feng, Hydrodynamic Interactions Among Bubbles, Drops, and Particles 551 in Non-Newtonian Liquids, Annu. Rev. Fluid Mech. 50 (2018) 505-534. doi:10.1146/annurev552 fluid-122316-045114. 
553 [41] M. Reiner, The Deborah Number, Phys. Today. 17 (1964) 62. doi:10.1063/1.3051374.

554 [42] R.P. Chhabra, Non-Newtonian Fluids: An Introduction, in: Rheol. Complex Fluids, 555 Springer Science \& Business Media, 2010: pp. 3-34.

556 https://link.springer.com/chapter/10.1007/978-1-4419-6494-6_1 (accessed February 22, 557 2019).

558 [43] M. Warshay, E. Bogusz, M. Johnson, R. C. Kintner, Ultimate velocity of drops in 559 stationary liquid media, Can. J. Chem. Eng. 37 (1959) 29-36. doi:10.1002/cjce.5450370107. 